\title{
Validation of combined Monte Carlo and photokinetic simulations for the outcome correlation analysis of benzoporphyrin derivative-mediated photodynamic therapy on mice
}

Karl W. Beeson Evgueni Parilov

Mary Potasek

Michele M. Kim

Timothy C. Zhu 


\title{
Validation of combined Monte Carlo and photokinetic simulations for the outcome correlation analysis of benzoporphyrin derivative-mediated photodynamic therapy on mice
}

\author{
Karl W. Beeson, ${ }^{a, \star}$ Evgueni Parilov, ${ }^{a}$ Mary Potasek, ${ }^{a}$ Michele M. Kim, ${ }^{\text {b }}$ and Timothy C. Zhu ${ }^{\text {b }}$ \\ ${ }^{a}$ Simphotek, Inc., Newark, New Jersey, United States \\ bUniversity of Pennsylvania, Department of Radiation Oncology, Philadelphia, Pennsylvania, United States
}

\begin{abstract}
We compare previously reported benzoporphyrin derivative (BPD)-mediated photodynamic therapy (PDT) results for reactive singlet oxygen concentration (also called singlet oxygen dose) on mice with simulations using a computational device, Dosie ${ }^{\mathrm{TM}}$, that calculates light transport and photokinetics for PDT in near real-time. The two sets of results are consistent and validate the use of the device in PDT treatment planning to predict BPD-mediated PDT outcomes in mice animal studies based on singlet oxygen dose, which showed a much better correlation with the cure index than the conventional light dose. $\odot$ The Authors. Published by SPIE under a Creative Commons Attribution 3.0 Unported License. Distribution or reproduction of this work in whole or in part requires full attribution of the original publication, including its DOI. [DOI: 10.1117/1.JBO.24.3.035006]
\end{abstract}

Keywords: medical device; photodynamic therapy; solid cancer therapy.

Paper 180572R received Oct. 18, 2018; accepted for publication Mar. 5, 2019; published online Mar. 14, 2019.

\section{Introduction}

Photodynamic therapy (PDT) has been approved by the U.S. Food and Drug Administration for the treatment of esophageal cancer, non-small cell lung cancer, Barrett's esophagus, and age-related macular degeneration. ${ }^{1-6}$ Two types of PDT killing mechanisms are known to exist when utilizing light-activated photosensitizers (PSs) to generate reactive oxygen species that kill cancer cells. Type I PDT generates superoxide anion $\left(\mathrm{O}_{2}{ }^{-} \cdot\right)$ and other secondary oxygen species, such as hydroxyl radicals ( $\mathrm{HO} \cdot)$ or hydrogen peroxide $\left(\mathrm{H}_{2} \mathrm{O}_{2}\right)$ for treatment. ${ }^{7}$ Many commonly used PSs are type II. Type II PDT is a complex process ${ }^{7}$ involving the interactions of the local photosensitizer concentration [PS], the local light fluence rate (intensity or $\phi$ ), and the local oxygen concentration $\left[{ }^{3} \mathrm{O}_{2}\right]$ in order to produce singlet oxygen $\left({ }^{1} \mathrm{O}_{2}\right)$. Furthermore, the local light fluence rate depends on light transmission through intervening tissue having local absorption coefficient, $\mu_{\mathrm{a}}$, and local reduced scattering coefficient, $\mu_{\mathrm{s}}^{\prime}$. The complexity of the type II PDT process has made monitoring of treatments difficult and unreliable. Many clinical studies have not resulted in optimal treatment due to the lack of reliable dosimetry for singlet oxygen. ${ }^{6}$

Several types of dosimetry have been utilized to measure the response of cancer to PDT, including incident light dose ${ }^{8}$ (or incident fluence, which equals incident fluence rate times the treatment time), PDT dose $^{9}$ (the time integral of PS concentration times the fluence rate), and singlet oxygen dose $\mathrm{s}^{10-12}$ (also denoted as reacted singlet oxygen concentration or $\left[{ }^{1} \mathrm{O}_{2}\right]_{r x}$ ). The most common PDT dosimetry method used by most PDT researchers and medical practitioners is the relatively simple procedure of measuring incident in-air fluence rather than using a more comprehensive method that accounts for patient-specific

*Address all correspondence to Karl W. Beeson, E-mail: beesonk@ simphotek .com in-vivo fluence distribution, which depends on tissue optical properties, photochemical parameters, treatment time, molecular oxygen concentration, etc. The drawback to the simplicity of incident fluence dosimetry is that treatment results for the same light dose can vary considerably for different patients.

PDT dose is a better alternative to light dose as it accounts not only to tissue light distribution but also to treatment/patientspecific unevenness of [PS]. Although ${ }^{1} \mathrm{O}_{2}$ is thought to be the major cause of cell toxicity for type II PSs, ${ }^{1} \mathrm{O}_{2}$ is very difficult to directly measure. However, the dosimetry quantity $\left[{ }^{1} \mathrm{O}_{2}\right]_{r x}$ can be calculated by combining photokinetics (PK) equations with calculations for light transport. ${ }^{13}$ This dosimetry quantity can then be used to predict treatment outcomes.

Modeling the photophysics/photochemistry of PDT is very challenging and a significant problem for improving the PDT treatments. Over the past several years, Zhu et al., ${ }^{9-13}$ Foster et al., ${ }^{14-17}$ Wilson et al., ${ }^{18,19}$ and other groups ${ }^{20-23}$ have proposed or described various PDT models to compute limited aspects of PDT, such as light transport, variation of optical parameters, and aspects of oxygen concentration and flow. In addition, over the past several years, there have been developments of treatment planning and monitoring tools using the laser light dose as the main criterion ${ }^{24-28}$ of PDT effectiveness but without the critical photophysics. For example, Davidson et al. ${ }^{29}$ developed a treatment planning software package and employed it in a phase II clinical trial of Tookad ${ }^{\mathrm{TM}}$-mediated PDT of persistent prostate carcinoma following radiation therapy. Treatment plans were based on pretreatment MRI images. Optical properties were determined by fitting a diffusion-based model to the in vivo fluence rate measurements. The treatment plan was evaluated by the light dose distribution superimposed on the MRI images of the largest volume. Light distribution calculations were verified by comparing fluence rate measurements made prior to Tookad $^{\mathrm{TM}}$ infusion, with fluence rate data extracted during treatment. Treatment results were measured six months post-treatment 
with biopsies. In patients where the light dose was less than $23 \mathrm{~J} / \mathrm{cm}^{2}$, none had a cancer-free biopsy response. In tumors treated with light dose greater than $23 \mathrm{~J} / \mathrm{cm}^{2}, 62 \%$ had cancer-free biopsies after six months. The dosimetry concentrated on the light optical properties of the tissue and the light fluence delivered to various regions of the prostate. Results were not conclusive based solely on light dosimetry. A phase I clinical trial of motexafin lutetium-mediated PDT of prostate cancer was performed at Penn. ${ }^{30-32}$ An integrated dosimetry and treatment planning system was developed at Penn utilizing a kernel-based algorithm to calculate light fluence rate distribution in a heterogeneous medium. ${ }^{33,34}$ The integrated system included a PDT dosimetry system to determine the 3-D distribution of tissue optical properties or diffuse optical tomography, ${ }^{35,36}$ as well as the distribution of PS concentration and oxygen saturation $\left(\mathrm{StO}_{2}\right){ }^{37}$ It utilized an optimization algorithm to determine the optimal positions of sources based on either light fluence ${ }^{38}$ or singlet oxygen. ${ }^{39}$ Preliminary results show that motexafin lutetium-mediated PDT will have an effect on the prostate-specific antigen (PSA) level when the light dose is larger than $150 \mathrm{~J} / \mathrm{cm}^{2}{ }^{32}$ PDT treatment planning has also been done using FullMonte tetrahedralbased MC software that may be more accurate if tissue surfaces are curved. ${ }^{40}$ Since the geometry for this study is planar, tetrahedral-based MC simulations are not expected to modify the results.

Commercial treatment planning and monitoring software, "Interactive Dosimetry by Sequential Evaluation" (iDOSE ${ }^{\circledR}$ ), was developed by Spectracure, Inc. It provided light dose plans with optical fiber positions based on three dimensional (3-D) tissue models generated from ultrasound. ${ }^{26}$ The software optimizes the fiber positions and provides a clinically acceptable plan for laser light in the tumor. A first monitoring sequence is performed after the optical fibers are in place. Initially, homogeneous optical properties are assumed for each cluster of optical fibers and initial monitoring is performed. At specific intervals, the light is interrupted and a monitoring evaluation test is performed. Tissue optical properties are obtained using the same fibers used for delivering the therapeutic light. This enables one to determine the effective attenuations and update the light dose. In a phase I/II clinical study, the Spectracure system was used with mTHPC $\left(\right.$ Foscan $\left.^{\circledR}\right)$ in the treatment of patients with histologically proven untreated, organ-confined prostate cancer. Initially, a conservative light dose of $5 \mathrm{~J} / \mathrm{cm}^{2}$ was used to limit damage to surrounding tissue. Following treatment, the PSA level was higher than expected for complete treatment and it was concluded that the light dose of $5 \mathrm{~J} / \mathrm{cm}^{2}$ was insufficient. In a later preclinical study, in male canines, this group suggested that the threshold light dose should be in the range of 20 to $30 \mathrm{~J} / \mathrm{cm}^{2}$ for effective PDT with mTHPC in the treatment of prostate cancer. However, iDOSE ${ }^{\circledR}$ does not include the PK of the PS and oxygen, which makes the treatment outcome dependent on a sufficient level and evenness of PS and oxygen concentrations.

These studies ${ }^{9-39}$ demonstrate the need for a more predictive, comprehensive, fast, and complete computer simulation for PDT. In this study, we expand on simulations developed at the University of Pennsylvania, Department of Radiation Oncology (Penn). ${ }^{9-13}$ The aim is to create a more complete simulation of PDT treatment that can be used for animal studies as well as in the clinic in near real-time. Simphotek, Inc. has developed Dosie ${ }^{\mathrm{TM}}$, an integrated proprietary computational device consisting of proprietary software and designated hardware. In the study presented here, we compare fluence rate and $\left[{ }^{1} \mathrm{O}_{2}\right]_{r x}$ results using Dosie ${ }^{\mathrm{TM}}$ to experimental and approximate simulations for superficial BPD-mediated PDT obtained by Kim et al. ${ }^{10}$ at Penn in animal studies. The Dosie ${ }^{\mathrm{TM}}$ device can also be used to simulate any PS for which the PK parameters are known. In the future, we plan to validate Dosie ${ }^{\mathrm{TM}}$ in clinical trials.

\section{Simulation Software and Designated Hardware}

\subsection{Integrated Computational Device}

The Dosie ${ }^{\mathrm{TM}}$ hardware and proprietary software incorporates, in one integrated computational device, Monte Carlo (MC) simulations of light transport, light fluence rate, and light dose (fluence) in target regions as well as PK simulations of singlet oxygen dose $\left(\left[{ }^{1} \mathrm{O}_{2}\right]_{r x}\right)$ and PDT dose. The MC approach is ideal for simulating light transport in biological tissue. ${ }^{41,42}$ This method has been greatly expanded over the years and has been successfully ported ${ }^{43}$ onto graphical processor units (GPU) to improve its performance. The subsequent PK simulations use the MC results and PS properties as inputs to calculate the resulting PDT dose and $\left[{ }^{1} \mathrm{O}_{2}\right]_{r x}$.

A schematic diagram of Dosie ${ }^{\mathrm{TM}}$ is shown in Fig. 1. The host process is an independent component of the software that is responsible for scheduling all PDT-related numerical calculations. It provides access to and controls two modules: light transport (MC-module) and PK-module. A graphical user interface (GUI) enables users to define PDT session simulation parameters, to launch and control simulations, and to visualize the output. The parameters needed for the numerical calculations are added from a database or from direct optical measurements before treatment. They include the 3-D target shape (prepared by third-party software, generated by measurements, such as CT or MRI), the optical parameters of the target (light scattering and light absorption coefficients), PK parameters for the PS (see Table 1 for BPD parameters), and the initial oxygen concentration. The variable parameters include laser power and location (s), PS (drug) concentration, and treatment time. The first calculation step is to use the MC-module to determine the light intensity (fluence rate) and light dose (fluence) at every microscopic point in the tumor. CUDA calculations are performed on a massively parallel GPU processor to simulate the paths of tens of millions of light rays. The calculated light intensity field is transferred from GPU memory to the host memory to launch the PK-module on the CPU processers to calculate the PDT PK that includes the light-PS-excitation (and photobleaching), the PS-oxygen excitation to generate singlet oxygen, and the singlet oxygen reaction with the target (including singlet-oxygen induced photobleaching). Comprehensive proprietary graphics, developed by Simphotek in the Dosie ${ }^{\mathrm{TM}}$ device, are used to visualize 2-D and 3-D outputs of the light fluence (light dose) and fluence rate, PDT dose, and singlet oxygen dose as well as concentrations of PS, ground-state oxygen, and cancer killing the $\left[{ }^{1} \mathrm{O}_{2}\right]_{r x}$ at every microscopic point in the 3 -D target for the duration of the treatment. This enables the researcher or physician to localize areas of possible undertreatment or overtreatment in the operating room while the patient is undergoing treatment and make corrections.

\subsection{MC-Module (Light Transport)}

The MC-module is based on MC photon transport that allows modeling light propagating in a heterogeneous translucent 


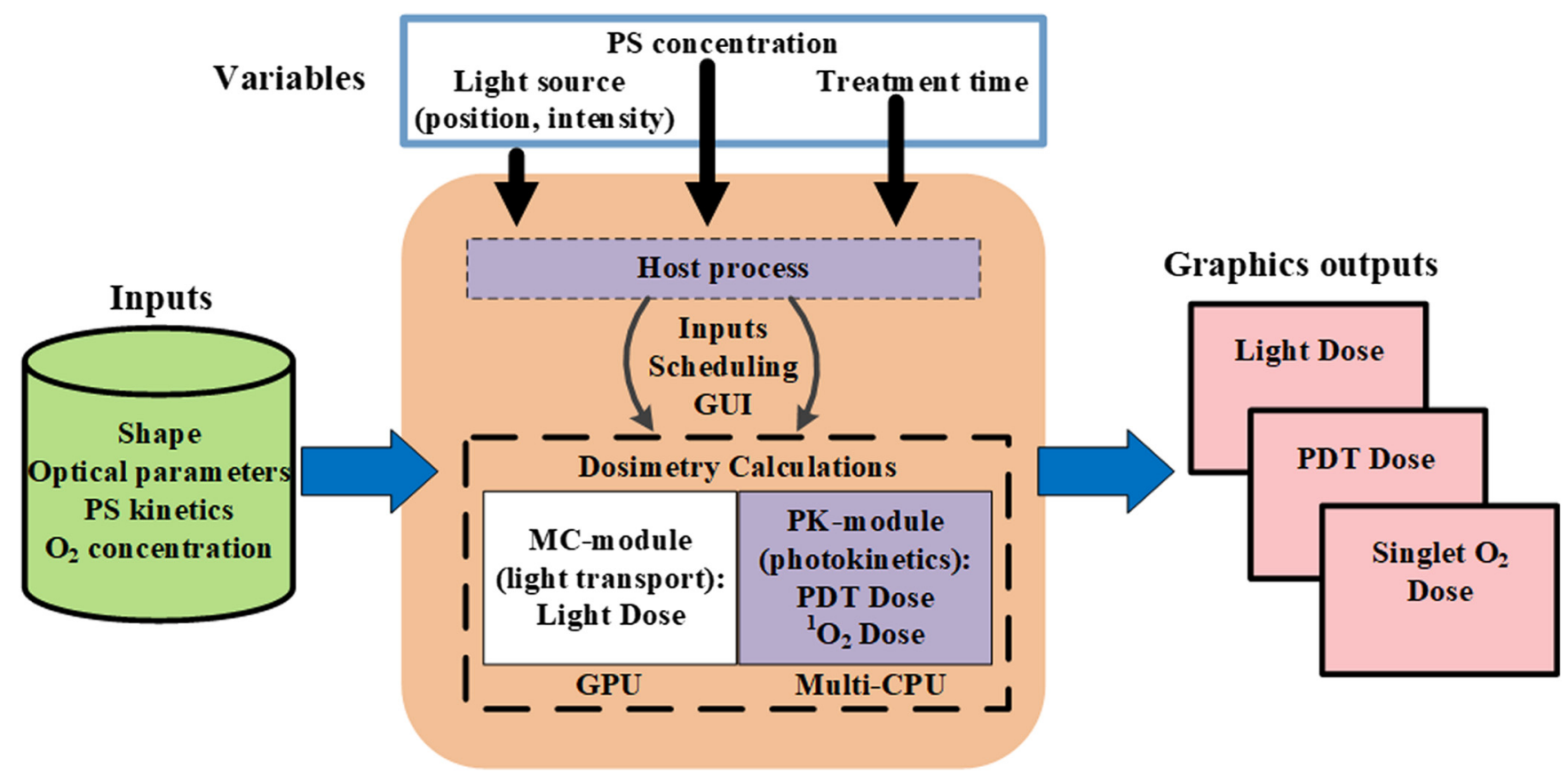

Fig. 1 Schematic diagram for Dosie ${ }^{\mathrm{TM}}$ integrated computer and software computational device. The computer includes both a GPU and a multicore CPU. The software first performs a MC simulation of light transport that is followed by a PK simulation.

Table 1 BPD simulation parameters. ${ }^{10,44}$

Simulation parameters for $\mathrm{BPD}^{\circledR}$

$$
\begin{aligned}
& g\left(\mu \mathrm{M} \mathrm{s}^{-1}\right)=1.7 \\
& \delta(\mu \mathrm{M})=33 \\
& \beta(\mu \mathrm{M})=11.9 \\
& \sigma\left(\mu \mathrm{M}^{-1}\right)=1.8 \times 10^{-5} \\
& \xi\left(\mathrm{cm}^{2} \mathrm{~mW}^{-1} \mathrm{~s}^{-1}\right)=0.055
\end{aligned}
$$

medium. Such a medium is characterized by high likelihood of (mostly forward) light scattering events occurring within short distances (submillimeter scale) and by a high albedo (absorption is typically two orders of magnitude smaller than the scattering). The MC-module numerically estimates the resulting 3-D maps of light fluence rates and light doses (fluences) within the PDT target translucent region (target volume) during a planned PDT session for a given arrangement of the incident or interstitially applied light sources.

For BPD simulation, a collimated light source is located in air and directed perpendicular to the surface of the target volume. Our modified proprietary version of MC models' light propagation within voxel-based geometry, where the target volume is subdivided into voxels (cubes of identical sizes) so that the graphical output 3-D map of Dosie ${ }^{\mathrm{TM}}$ assigns one fluence rate value for each voxel. The target volume consists of heterogeneous media with known optical properties (i.e., absorption coefficient, scattering coefficient, scattering anisotropy factor, and refractive index) so that each voxel can be assigned a unique set of properties.
During MC simulations, millions of photon packets are launched toward the target volume, according to the light excitation distribution. Each packet-we will be using word "photon" instead for the rest of this section-has an initial weight of 1. Specular reflection/refraction is taken into account at the air-target surface interface by applying the Fresnel model for unpolarized light with the refractive index 1.4. Each photon can undergo multiple scattering events in the target volume until the photon either escapes the volume or is absorbed within the volume when its weight drops below a specified threshold. Within the medium, a photon propagates free, voxel by voxel, till the next scattering event. The length of such free propagation is randomly determined according to the scattering coefficient $\mu_{\mathrm{s}}$. If the optical properties change while the photon travels from one voxel to another, the free path is properly adjusted. At each scattering event, a trajectory direction is determined by applying the Henyey-Greenstein phase function. The photon weight is gradually reduced while traveling from one voxel to another by a factor $e^{-\mu_{a} l}$, where $l$ is the portion of its trajectory within each such a voxel. All fractions of the weight lost that occurred during propagation of each photon are deposited at every voxel it passes so that by the end of the simulation, we can estimate the fluence from the accumulated weights.

A voxel-based implementation of MC is relatively straightforward, as it does not require adding potentially time-consuming ray-boundary intersection tests. However, for geometries with multiple curved surfaces and/or for less scattering media, a boundary-based $\mathrm{MC}$ implementation may result in more accurate solutions due to more precise reflection/refraction calculations along the boundaries (e.g., as in this tetrahedral-based ${ }^{40} \mathrm{MC}$ implementation).

MC simulations can be very time consuming (i.e., hours) for translucent media. The main challenge is to make the simulations run in near real-time (i.e., a minute or less). At the current state of the computer hardware, only parallel calculations are 
capable of reaching near real-time performance. MC-proprietary module software in Dosie ${ }^{\mathrm{TM}}$ is a GPU CUDA code (an extension to $\mathrm{C}++$ programming language that allows scheduling identical compute kernels to run as threads on GPU Core processors in parallel; CUDA compute capability 5.0 has been used in Dosie ${ }^{\mathrm{TM}}$ ) with a performance target of completing target light transport simulations within minutes. Each CUDA thread performs a simulation of a photon propagation accessing the voxels' optical properties of each encountered voxel from the device constant memory and depositing the weight's losses to the device shared memory. The final fluence calculations are done in the host process after the accumulated weights are transferred from the device memory to the host memory using the proprietary Dosie ${ }^{\mathrm{TM}}$ device, including experimental hardware configuration of Core i7-6820HQ (16G memory, 64-bit) CPU and NVIDIA Quadro M2000M (640 cores, 4G memory, CUDA 5.0) GPU, the near-real time target performance has been achieved.

\section{$2.3 \quad$ PK-Module}

The PK-module of Dosie ${ }^{\mathrm{TM}}$ is a complicated and essential part of modeling PDT. Unlike the light transport, this is a quasiquantum mechanical process in which the laser light energy (photon) is transformed into reactive chemical agents that kill cancer cells. The PK calculations are done for each voxel independent from other voxels. This allows the calculations to run in parallel by taking advantage of a multicore CPU architecture. PK Eqs. (1)-(3) are used to calculate the time evolution of the (ground state) PS concentration, $\left[S_{0}\right]$, the ground state oxygen concentration, $\left[{ }^{3} \mathrm{O}_{2}\right]$, and the reacted singlet oxygen concentration, $\left[{ }^{1} \mathrm{O}_{2}\right]_{r x}$, where the value $\left[S_{0}\right]$ is the BPD concentration and $\phi$ is the fluence rate. This is an initial value problem with the given initial fields $\left[S_{0}\right](x, y, z, t=0) \geq 0$ and $\left[{ }^{3} \mathrm{O}_{2}\right](x, y, z, t=$ $0)>0$ while $\left[{ }^{1} \mathrm{O}_{2}\right]_{r x}(x, y, z, t=0)=0$, and with satisfying a non-negativity requirement at all times: $\left[S_{0}\right](t>0) \geq 0$ and $\left[{ }^{3} \mathrm{O}_{2}\right](t>0) \geq 0$. The initial $\left[S_{0}\right]$ for each mice group is listed in Table 2. A variant of an embedded Runge-Kutta formulation RK5(4), adjusted to satisfy a non-negativity condition on a solution, is implemented in PK-module as a numerical proprietary $\mathrm{C}++$ code to solve the system Eqs. (1)-(3). Previously, Penn solved $^{10,13}$ a similar system using MATLAB internal solver. Simphotek's PK-module has been validated against MATLAB calculations ${ }^{45}$ resulting in a perfect match for the published parameters. The equations are as follows: ${ }^{10,13}$

$$
\frac{d\left[S_{0}\right]}{d t}+\left(\xi \sigma \frac{\phi\left(\left[S_{0}\right]+\delta\right)\left[{ }^{3} O_{2}\right]}{\left[{ }^{3} O_{2}\right]+\beta}\right)\left[S_{0}\right]=0,
$$

Table 2 Summary of the results for all mice groups. Penn data are taken from Ref. 10. The optical power (column 6) used for the Dosie ${ }^{\mathrm{TM}}$ simulations equals the area of the $10-\mathrm{mm}$ diameter emitting disc $\left(0.7854 \mathrm{~cm}^{2}\right)$ times the Penn in-air fluence rate $(\mathrm{column} 2)$. The $\left[{ }^{1} \mathrm{O}_{2}\right]_{r x}$ simulated results for $1.00-, 0.50-$ and $0.25-\mathrm{mm}$ voxels are shown in columns 8,9 , and 10 , respectively. The values in parentheses for columns 8,9 , and 10 are the mismatch to the Penn values (column 7).

\begin{tabular}{|c|c|c|c|c|c|c|c|c|c|c|c|}
\hline 1 Penn & 2 Penn & 3 Penn & 4 Penn & 5 Penn & 6 Dosie $^{\mathrm{TM}}$ & 7 Penn & 8 Dosie $^{\mathrm{TM}}$ & 9 Dosie $^{\mathrm{TM}}$ & 10 Dosie $^{\mathrm{TM}}$ & 11 Penn & 12 Penn \\
\hline Group & $\begin{array}{c}\text { Incident } \\
\text { in-air } \\
\text { fluence rate } \\
\left(\mathrm{mW} / \mathrm{cm}^{2}\right)\end{array}$ & $\begin{array}{c}\text { Time } \\
\text { (s) }\end{array}$ & $\begin{array}{l}\text { Incident } \\
\text { in-air } \\
\text { fluence } \\
\left(\mathrm{J} / \mathrm{cm}^{2}\right)\end{array}$ & $\begin{array}{c}{[\mathrm{BPD}]_{\text {pre }}} \\
(\mu \mathrm{M})\end{array}$ & $\begin{array}{c}\text { Power } \\
\text { in } \\
10 \mathrm{~mm} \\
\text { disc }(\mathrm{W})\end{array}$ & $\begin{array}{c}\text { At } 3 \mathrm{~mm} \\
\text { depth } \\
{\left[{ }^{1} \mathrm{O}_{2}\right]_{r x}} \\
(\mathrm{mM})\end{array}$ & $\begin{array}{c}\qquad\left[{ }^{1} \mathrm{O}_{2}\right]_{r x} \\
(\mathrm{mM}) \\
1.00 \mathrm{~mm} \\
\text { grid (\% diff.) }\end{array}$ & $\begin{array}{c}\left.\qquad{ }^{1} \mathrm{O}_{2}\right]_{r x} \\
(\mathrm{mM}) \\
0.50 \mathrm{~mm} \\
\text { grid (\% diff.) }\end{array}$ & $\begin{array}{c}{\left[{ }^{1} \mathrm{O}_{2}\right]_{r x}} \\
(\mathrm{mM}) \\
0.25 \mathrm{~mm} \\
\text { grid (\% diff.) }\end{array}$ & $\begin{array}{c}K \\
\text { (1/days) }\end{array}$ & $\begin{array}{l}\text { Index, } \\
\mathrm{Cl}\end{array}$ \\
\hline 1 & 50 & 600 & 30 & 0.53 & 0.0393 & 0.39 & $0.4067(+4.29)$ & $0.4057(+4.04)$ & $0.4054(+3.95)$ & 0.40 & 0.0377 \\
\hline 2 & 75 & 400 & 30 & 0.72 & 0.0589 & 0.45 & $0.4607(+2.39)$ & $0.4633(+2.95)$ & $0.4638(+3.06)$ & 0.38 & 0.0556 \\
\hline 3 & 150 & 200 & 30 & 0.56 & 0.1178 & 0.29 & $0.2913(+0.46)$ & $0.2936(+1.24)$ & $0.2941(+1.43)$ & 0.40 & 0.0237 \\
\hline 4 & 50 & 1400 & 70 & 0.73 & 0.0393 & 0.90 & $0.9147(+1.63)$ & $0.9200(+2.23)$ & $0.9213(+2.36)$ & 0.28 & 0.3151 \\
\hline 5 & 75 & 1333 & 100 & 0.41 & 0.0589 & 0.60 & $0.5977(-0.39)$ & $0.6032(+0.53)$ & $0.6044(+0.74)$ & 0.37 & 0.1037 \\
\hline 6 & 50 & 2700 & 135 & 0.50 & 0.0393 & 0.78 & $0.7839(+0.50)$ & $0.7910(+1.41)$ & $0.7928(+1.64)$ & 0.34 & 0.1646 \\
\hline 7 & 75 & 1800 & 135 & 0.53 & 0.0589 & 0.82 & $0.8246(+0.56)$ & $0.8319(+1.45)$ & $0.8337(+1.67)$ & 0.32 & 0.2139 \\
\hline 8 & 150 & 900 & 135 & 0.58 & 0.1178 & 0.85 & $0.8626(+1.48)$ & $0.8684(+2.17)$ & $0.8698(+2.33)$ & 0.28 & 0.3240 \\
\hline 9 & 75 & 2000 & 150 & 0.84 & 0.0589 & 1.30 & $1.3094(+0.72)$ & $1.3196(+1.51)$ & $1.3222(+1.71)$ & 0 & 1 \\
\hline 10 & 100 & 1500 & 150 & 0.66 & 0.0785 & 1.03 & $1.0292(-0.07)$ & $1.0373(+0.70)$ & $1.0393(+0.90)$ & 0.11 & 0.7432 \\
\hline 11 & 75 & 3333 & 250 & 0.58 & 0.0589 & 0.96 & $0.9600(+0.00)$ & $0.9630(+0.31)$ & $0.9637(+0.38)$ & 0.25 & 0.3878 \\
\hline 12 & 150 & 1667 & 250 & 0.77 & 0.1178 & 1.26 & $1.2599(-0.01)$ & $1.2651(+0.40)$ & $1.2664(+0.50)$ & 0 & 1 \\
\hline 13 & 150 & 2000 & 300 & 0.77 & 0.1178 & 1.27 & $1.2728(+0.22)$ & $1.2758(+0.46)$ & $1.2766(+0.52)$ & 0 & 1 \\
\hline 14 & 150 & 2333 & 350 & 0.81 & 0.1178 & 1.35 & $1.3432(-0.50)$ & $1.3450(-0.37)$ & $1.3454(-0.34)$ & 0 & 1 \\
\hline 15 & 0 & 0 & 0 & & & & & & & 0.41 & 0 \\
\hline
\end{tabular}


$\frac{d\left[{ }^{3} O_{2}\right]}{d t}+\left(\xi \frac{\phi\left[S_{0}\right]}{\left.{ }^{3} O_{2}\right]+\beta}\right)\left[{ }^{3} O_{2}\right]-g\left(1-\frac{\left[{ }^{3} O_{2}\right]}{\left.{ }^{3} O_{2}\right](t=0)}\right)=0$,

$\frac{d\left[{ }^{1} O_{2}\right]_{r x}}{d t}-\left(\xi \frac{\phi\left[S_{0}\right]\left[{ }^{3} O_{2}\right]}{\left[{ }^{3} O_{2}\right]+\beta}\right)=0$.

In these equations, $g$ is the oxygen supply rate to the tissue, $\delta$ is the low-concentration correction, $\beta$ is the oxygen quenching threshold concentration, $\sigma$ is the specific photobleaching ratio, and $\xi$ is the macroscopic maximum oxygen supply rate. The parameter values specific for the PS, BPD, are given in Table 1. The initial oxygen concentration, $\left[{ }^{3} \mathrm{O}_{2}\right](x, y, z$, $t=0)$, is assumed to be $40 \mu \mathrm{M}$ for all simulations.

\subsection{Graphical User Interface}

The calculations are broken into individual sessions. Using the GUI created in Dosie ${ }^{\mathrm{TM}}$, users can create sessions, archive current sessions, browse and search for previous sessions, define PDT simulation parameters, and launch simulations. The visualization component in Dosie ${ }^{\mathrm{TM}}$ includes tools to render 2-D and 3-D simulation and geometry data. It enables users to visualize and analyze dose maps resulting from MC and PK calculations (see Fig. 2) to visually inspect under- and overdosed regions. Visualization view has a control panel that lists all dose maps currently available for visualization for each completed session or allows uploading maps from the file system and creating groups of maps for intersession analysis.

The GUI shows four conventional views for visualization of the target data: one model view with a 3-D map and three 2-D slice views of the individual 2-D slices resulting in a 2-D sagittal (side) view, a 2-D coronal (front) view, and a 2-D transverse (horizontal) view. The slicing planes can be moved to visualize different cross-sections of the 3-D calculations shown in model view. This enables the user to investigate the fine, near microscopic details of the treatment in the target.

In this study, we will focus on the MC fluence rate and $\left[{ }^{1} \mathrm{O}_{2}\right]_{r x}$ singlet oxygen dose- the quantity that showed a strong correlation with PDT outcome in recent animal studies. ${ }^{10}$ The voxel-based MC simulations of light transport run over $100 \times$ $100 \times 100$ grids of either $(1 \mathrm{~mm})^{3},(0.5 \mathrm{~mm})^{3}$, or $(0.25 \mathrm{~mm})^{3}$ voxels. From the resulting fluence rate maps, the subsequent PK calculations determine the singlet oxygen dose or $\left[{ }^{1} \mathrm{O}_{2}\right]_{r x}$ for each voxel. We show that Dosie ${ }^{\mathrm{TM}}$ and the Penn preliminary calculations give nearly equivalent results.

\section{Experimental and Computational Methods}

\subsection{Experimental Procedures Summary}

Superficial PDT was done at Penn using mice having radiationinduced fibrosarcoma (RIF) tumors. The cancerous mice were injected with the PS benzoporphyrin derivative (BPD, Visudyne ${ }^{\circledR}$ ) followed by PDT treatments. ${ }^{10}$ The authors define a cure index for the mice and analyze the correlation of the cure index to the three types of doses: light fluence dose, PDT-dose, and singlet oxygen dose. Penn denotes the singlet oxygen dose as the reacted singlet oxygen concentration, or $\left[{ }^{1} \mathrm{O}_{2}\right]_{r x}$.

Penn researchers divided the mice into 15 groups, each containing 3 to 5 mice. PDT was delivered to 14 groups. The 15 th group was the control group that did not receive either BPD or PDT. RIF cells were cultured and $30 \mu 1$ (at $1 \times 10^{7}$ cells $/ \mathrm{ml}$ )
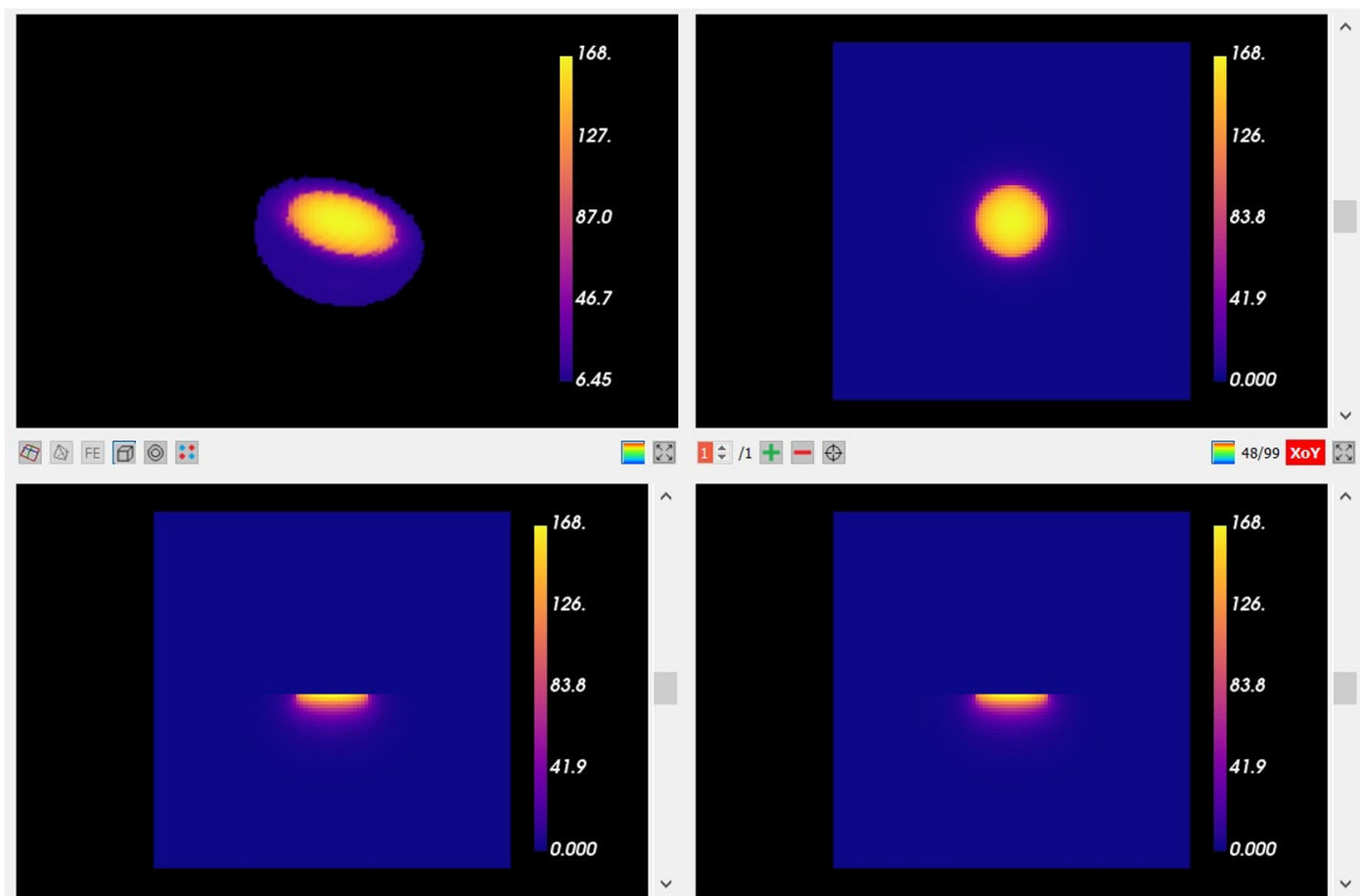

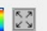
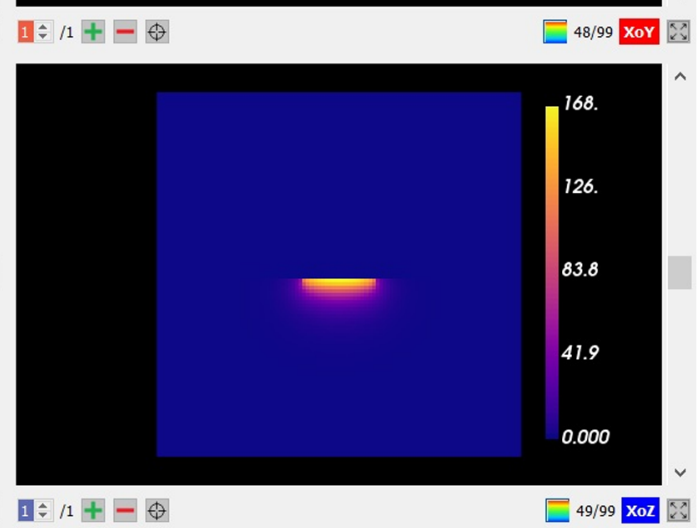

Fig. 2 Dosie $^{\mathrm{TM}} \mathrm{GUI}$ screenshots: MC fluence rate results using 0.50-mm voxels for an incident fluence rate of $50 \mathrm{~mW} / \mathrm{cm}^{2}$. Volume rendering is done to visualize 3-D fluence rate map in the upper left view, whereas other views show three 2-D cross-sections (slices) of the map-sagittal, coronal, and transverse views. 
were injected in the right shoulders of 6- to 8-week old female $\mathrm{C} 3 \mathrm{H}$ mice. The resulting tumors were treated with PDT when the tumors were $\sim 3$ to $5 \mathrm{~mm}$ in diameter and $\sim 3$ - $\mathrm{mm}$ thick. The mice were injected with BPD $3 \mathrm{~h}$ before treatment. The mice were then treated with a $10-\mathrm{mm}$ diameter, $690 \mathrm{~nm}$, collimated light beam from an $8 \mathrm{~W}$ diode laser (B\&W Tek Inc., Newark, Delaware). The incident in-air fluence was 30 to $350 \mathrm{~J} / \mathrm{cm}^{2}$, which corresponded from 50 to $150 \mathrm{~mW} / \mathrm{cm}^{2}$ fluence rate.

Simulations of singlet oxygen dose require knowledge of the BPD concentration in the tumor, the optical properties of the tumor, and the initial ground-state oxygen concentration, $\left[{ }^{3} \mathrm{O}_{2}\right]$, in the tumor. Penn did fluorescence measurements on each mouse using a custom multifiber spectroscopic contact probe before treatment to determine the initial BPD concentration. ${ }^{46}$ The average initial BPD concentrations, $[\mathrm{BPD}]_{\text {pre }}$, for each group of mice, are listed in Table 2. Tissue optical properties for the tumors, $\mu_{\mathrm{a}}$ and $\mu_{\mathrm{s}}^{\prime}$, were also measured using a multifiber contact probe. ${ }^{44}$ For the simulations, it is assumed that the tissue has a single set of optical properties, $\mu_{\mathrm{a}}=0.69 \mathrm{~cm}^{-1}$ and $\mu_{\mathrm{s}}^{\prime}=11 \mathrm{~cm}^{-1}$, which are typical values for tumors in all mice groups.

To determine a cure index, CI, Penn measured tumor dimensions daily for 14 days after PDT and calculated tumor volumes using the formula: ${ }^{47} V=\pi a^{2} b / 6$, where $a$ and $b$ are diameters of the width and length axes. A tumor regrowth rate for each tumor was determined by the best fit to an exponential of the form, $e^{k t}$, where $t$ is the time (in units of days) after PDT. Then CI is obtained as follows: ${ }^{10}$

$\mathrm{CI}=1-\frac{k}{k_{\mathrm{ctr}}}$,

where $k$ is the tumor regrowth rate and $k_{\mathrm{ctr}}$ is the regrowth rate for the control group that was not injected with BPD and did not undergo light illumination. Penn results for CI are listed in Table 2.

\subsection{Calculations of Fluence Rate and $\left[{ }^{1} \mathrm{O}_{2}\right]_{r x}$}

The bulk of Penn simulations have been performed in MATLAB. To determine fluence rate versus tissue depth at the center of the light beam, Penn used the previously determined, one-dimensional (1-D) analytical expression to approximate MC results at the center of a $10-\mathrm{mm}$ diameter treatment light beam: ${ }^{48}$

$$
\frac{\phi}{\phi_{\text {air }}}=\left(1-b e^{-\lambda_{1} d}\right)\left(C_{2} e^{-\lambda_{2} d}+C_{3} e^{-\lambda_{3} d}\right),
$$

where the parameters $\lambda_{1}, \lambda_{2}, \lambda_{3}, b, C_{2}$, and $C_{3}$ for a $10-\mathrm{mm}$ diameter beam are functions ${ }^{48}$ of $\mu_{\mathrm{a}}, \mu_{s}^{\prime}, u_{\mathrm{eff}}$, and $R_{d}\left(R_{d}\right.$ is the diffuse reflectance at the interface between air and tissue). For $\mu_{\mathrm{a}}=0.69 \mathrm{~cm}^{-1}$ and $\mu_{\mathrm{s}}^{\prime}=11 \mathrm{~cm}^{-1}$, then $R_{d}=0.321$.

Full 3-D MC simulations of fluence rate versus depth were done for the same mice data using MC-module of Dosie ${ }^{\mathrm{TM}}$. The 3-D simulations of Dosie ${ }^{\mathrm{TM}}$ allow the visualization of the results in all three dimensions rather than just the 1-D results obtained by Penn only at the center of the treatment beam. Each MC simulation launched 20 million collimated light rays directed perpendicular to the target surface from a 10-mm-diameter disc (the area is $0.7854 \mathrm{~cm}^{2}$ ).

\section{Results}

\subsection{Fluence Rate Calculations}

In Figs. 2 and 3, we show Dosie ${ }^{\mathrm{TM}}$ 's partial screenshots of $\mathrm{MC}$ light transport results for the fluence rates inside the target volume using $0.50 \mathrm{~mm}$ voxels, $(0.50 \mathrm{~mm})^{3}$ cubes, an incident fluence rate of $50 \mathrm{~mW} / \mathrm{cm}^{2}$ assumes that the tissue has a single set of optical properties, $\mu_{\mathrm{a}}=0.69 \mathrm{~cm}^{-1}$ and $\mu_{\mathrm{s}}^{\prime}=11 \mathrm{~cm}^{-1}$. The Dosie ${ }^{\mathrm{TM}} \mathrm{MC}$ runtime was $21 \mathrm{~s}$ for 20 million photons.

There are four views in the MC output graphics in Fig. 2. In the upper left, a portion of the target volume with significant fluence values is rendered. The view is interactive allowing the user to rotate and magnify the target volume. In this example, the volume is tilted to view, where the light is incident on the target surface. The other three views are an XY slice (upper right), an YZ slice (lower left), and an XZ slice (lower right). The positions of each slice can be moved by the user within the target volume. Each slice can be expanded to fill the graphical image space. The graphics showing the expanded YZ slice (with added labels) of fluence rate values is shown in Fig. 3.

Note that although the incident fluence rate is $50 \mathrm{~mW} / \mathrm{cm}^{2}$, the peak fluence rate induced just below the surface is $168 \mathrm{~mW} /$ $\mathrm{cm}^{2}$ (as shown on the scale at the right), which is 3.36 times larger than the incident fluence rate. This enhanced fluence rate occurring just under the surface of the target volume is typical for scattering media, where light can be scattered in all directions and can undergo total internal reflection under the target surface, which adds significantly to the magnitude of the primary incident light.

A comparison of the analytical formula, Eq. (5), to MC calculations of Dosie ${ }^{\mathrm{TM}}$ for the ratio (fluence rate in tissue)/ (incident fluence rate in air) versus depth in tissue is shown in Fig. 4. For MC, the results at the center of the beam using $1.0,0.5$, and $0.25 \mathrm{~mm}$ grid steps are done for an incident fluence rate of $50 \mathrm{~mW} / \mathrm{cm}^{2}$. For the $1.0-\mathrm{mm}$ grid, the first voxel (centered at $0.5 \mathrm{~mm}$ depth) misses the initial higher ratio of fluences at $\sim 0.25 \mathrm{~mm}$ depth in the target. The $0.5-\mathrm{mm}$ and $0.25-\mathrm{mm}$ grids more accurately resolve the fluence ratios for the smaller depths. Consequently, the MC results for such grids are approximately identical to the analytical approximation.

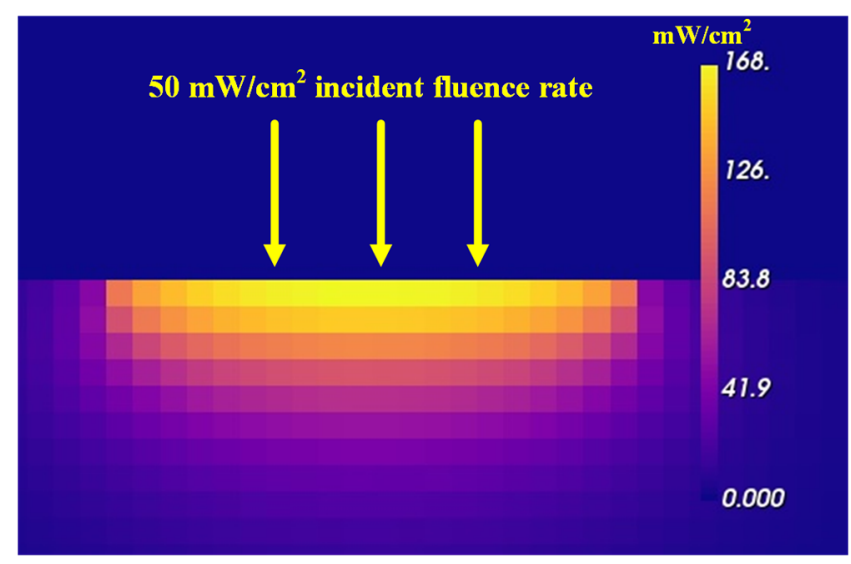

Fig. $3 \mathrm{YZ}$ slice of Dosie ${ }^{\mathrm{TM}}$ 's MC fluence rate results using 0.50-mm voxels through the center of the target volume for an incident fluence rate of $50 \mathrm{~mW} / \mathrm{cm}^{2}$. 


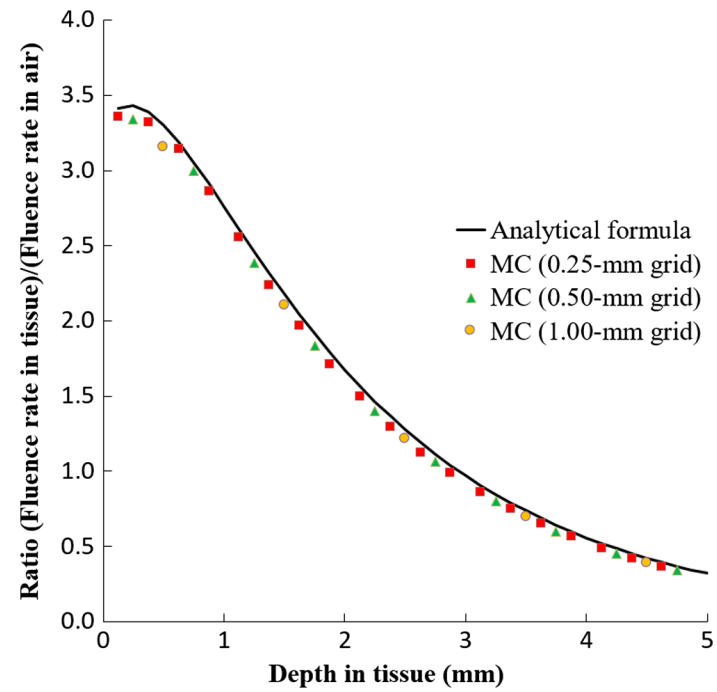

Fig. 4 The ratio (fluence rate in tissue)/(fluence rate in air) versus depth in tissue that compares an analytical fit ${ }^{48}$ to Dosie ${ }^{\text {TM's }}$ MC results.

\subsection{PK Calculations}

After the MC calculation of the fluence rate for each voxel of the target, the PK-module performs PK numerical calculations to find $\left[{ }^{1} \mathrm{O}_{2}\right]_{r x}$ for each such voxel of the target volume for a treatment time of $600 \mathrm{~s}$. The YZ slice at the center of the full 3-D simulation is shown in Fig. 5. The maximum $\left[{ }^{1} \mathrm{O}_{2}\right]_{r x}$ just below the surface at the center of the beam is $711 \mu \mathrm{M}$ or $0.711 \mathrm{mM}$. The Dosie ${ }^{\mathrm{TM}} \mathrm{PK}$ runtime was $12 \mathrm{~s}$ using 4 CPU processor cores.

To insure each tumor gets a sufficient dose through the full 3-mm tumor thickness, we determined the singlet oxygen dose at a distance of $3 \mathrm{~mm}$ below the surface of the target at the center of the illumination. For PK simulations with a 1-mm grid, one needs to look at the third and fourth layers of 1-mm-thick voxels at the center of illumination and to average the two results to get the singlet oxygen dose $3 \mathrm{~mm}$ below the target surface. For a $0.50-\mathrm{mm}$ grid, as shown in Fig. 5, this was done by looking at the sixth and seventh layers and by averaging the two results to get the singlet oxygen dose at the interface $3 \mathrm{~mm}$ below the target surface. For a $0.25-\mathrm{mm}$ grid, this was done by averaging the values from the 12th and 13th layers.

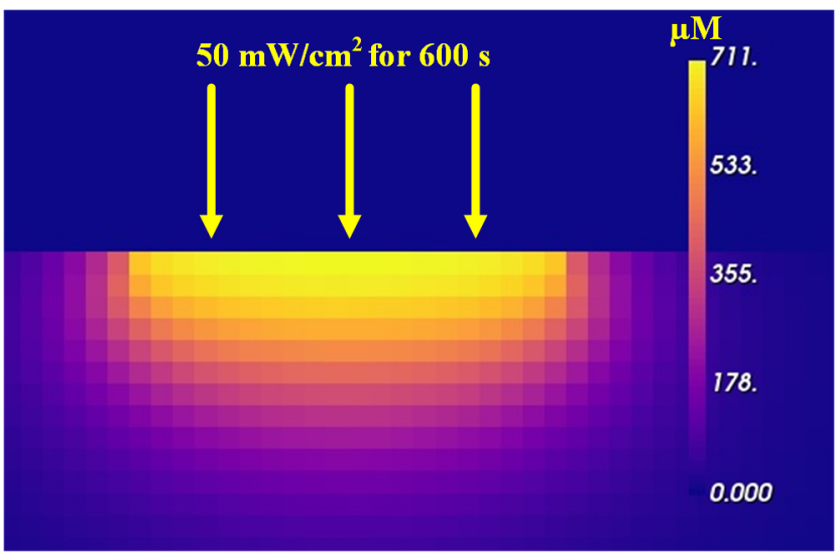

Fig. $5 \mathrm{YZ}$ slice of Dosie ${ }^{\mathrm{TM}}$ 's $\mathrm{PK}\left[{ }^{1} \mathrm{O}_{2}\right]_{r x}$ results through the center of the target volume shown for an incident fluence rate of $50 \mathrm{~mW} / \mathrm{cm}^{2}$ for $600 \mathrm{~s}$ and for $0.50-\mathrm{mm}$ voxels.

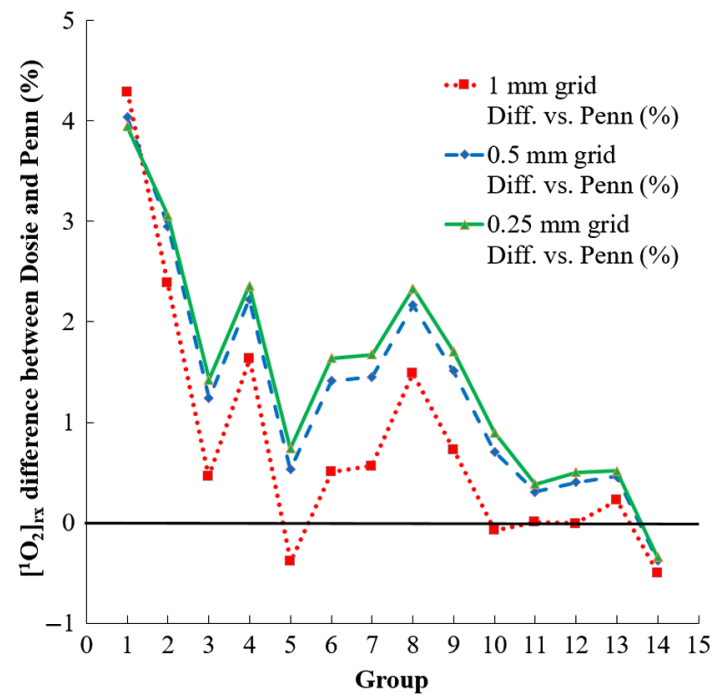

Fig. 6 Comparison of Dosie ${ }^{\text {TM's }}$ PK results to Penn results for 1-mm, $0.50-\mathrm{mm}$, and $0.25-\mathrm{mm}$ voxels.

The final Penn and Dosie ${ }^{\mathrm{TM}}$ simulation results for all the mice groups are shown in Table 2. Columns 8, 9, and 10 include Dosie $^{\mathrm{TM}}$ PK calculated values of $\left[{ }^{1} \mathrm{O}_{2}\right]_{r x}$ at $3 \mathrm{~mm}$ below for different grid resolutions: $1.0,0.5$, and $0.25 \mathrm{~mm}$ voxels, respectively. The numbers in parentheses show the mismatch with the corresponding Penn results (see column 7). Except for mice groups 1 and 2, the differences between the PK results and the Penn results are less than about $2 \%$.

Plots showing the differences between the PK results and the Penn results are shown in Fig. 6. In going from 1.00-mm voxels to $0.50-\mathrm{mm}$ voxels, the differences with the Penn results change on the order of $1 \%$. However, in going from $0.50-\mathrm{mm}$ voxels to 0.25 -mm voxels, the differences only change $\sim 0.2 \%$, indicating that reducing the grid step below $0.50 \mathrm{~mm}$ is unnecessary.

\section{Discussion}

To determine whether the singlet oxygen dose is a better PDT outcome predictor than the conventional light dose, we plot the cure index versus reacted singlet oxygen values $\left[{ }^{1} \mathrm{O}_{2}\right]_{r x}$, which are calculated by Dosie ${ }^{\mathrm{TM}}$ at $3 \mathrm{~mm}$ below the surface (Fig. 7), and versus the incident in-air fluence (Fig. 8). The cure index data are taken from the last column of Table 2 .

Figure 7 shows a strong correlation of CI with singlet oxygen dose. Note that $\mathrm{CI}=1.0$ (i.e., cure) indicates no tumor regrowth during the 14 days of the tumor monitoring. Furthermore, Fig. 7 shows that a threshold dose of $\left[{ }^{1} \mathrm{O}_{2}\right]_{r x}$ of $\sim 1.3 \mathrm{mM}$ is required in order to achieve a $\mathrm{CI}=1.0$. Significantly, lower singlet oxygen doses can allow the tumors to quickly regrow.

Figure 8 shows the poor correlation of $\mathrm{CI}$ with incident in-air fluence on the target surface. For example, for a narrow domain of incident fluence values of $135-150 \mathrm{~J} / \mathrm{cm}^{2}$, the CI values ranged from a poor value of about 0.16 to an excellent value of 1.0, indicating low correlation with fluence. Moreover, a good outcome has been observed for the incident fluence ranging from 150 to $350 \mathrm{~J} / \mathrm{cm}^{2}$, which makes it impossible to localize a "safe" range to use for the light dose during pretreatment planning. Singlet oxygen dose clearly shows a better correlation.

At this time, we can suggest some possible additional applications of Dosie ${ }^{\mathrm{TM}}$. For example, it may be useful for 


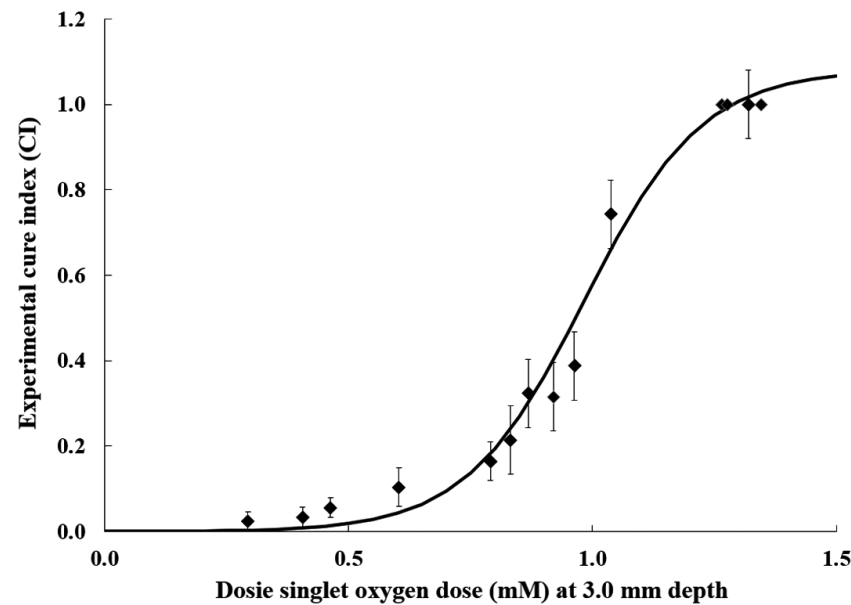

Fig. 7 Penn experimental cure index $(\mathrm{Cl})$ data from Ref. 10 versus Dosie $^{\mathrm{TM}}$ calculated singlet oxygen dose, $\left[{ }^{1} \mathrm{O}_{2}\right]_{r x}$, at $3 \mathrm{~mm}$ below the target surface for $0.50-\mathrm{mm}$ grid. The $\mathrm{Cl}$ error bars and the expression for the solid line, $\mathrm{Cl}=1.08 /\left(1+3490 \times \exp \left(-8.301 \times\left[{ }^{1} \mathrm{O}_{2}\right]_{r x}\right)\right)$ are from Ref. 10.

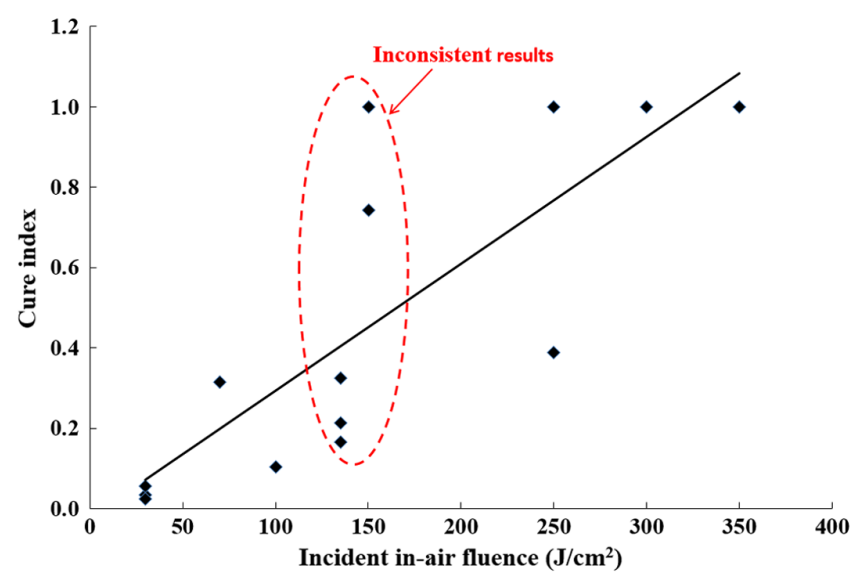

Fig. 8 Cure index versus conventional light dose (in-air incident fluence on the target surface). Data taken from Ref. 10.

designing and trying-out preclinical studies (i.e., animal trials), thus reducing the time and costs associated with expensive trials that can cost hundreds-of-thousands of dollars and months or years. One could use Dosie ${ }^{\mathrm{TM}}$ to try hundreds of possibilities before incurring major costs. Additionally, Dosie ${ }^{\mathrm{TM}}$ may be used for certain superficial clinical cancer treatments in research hospitals, for cancers such as esophagus, endobronchial, oral cavity, or skin. Research hospitals are performing clinical studies in esophagus (Mem. Sloan-Kettering, New York, NCT03133650), oral cavity (Roswell Park Cancer Institute, New York, NCT02119728), and certain skin conditions (Cleveland Clinic, Ohio, NCT 02124733).

\section{Conclusion}

PDT treatment results depend on many variables, such as tissue oxygenation, PS concentration, tissue optical parameters, PK parameters, incident fluence rate, and treatment time. A reliable and accurate dosimetry method that takes these variables into account is needed in order to make PDT outcomes predictable. The Dosie ${ }^{\mathrm{TM}}$ simulation results in this study are consistent with Penn results ${ }^{10}$ that validate the use of Dosie ${ }^{\mathrm{TM}}$ to predict BPDmediated PDT results on mice animal studies. Furthermore, the results indicate that calculated singlet oxygen dose, $\left[{ }^{1} \mathrm{O}_{2}\right]_{r x}$, is a very good predictor of PDT outcomes, whereas incident in-air fluence - the conventional light dose-may be a poor predictor. The results indicate that planning and monitoring of PDT treatments should preferably utilize simulations of singlet oxygen dose rather than light dose in order to better predict and improve treatment outcomes.

\section{Disclosures}

None of the authors have a financial conflict of interest in this work.

\section{Acknowledgments}

This work was supported by an SBIR grant from the National Cancer Institute of the National Institutes of Health under Grant No. R44CA183236.

\section{References}

1. Z. Huang, "A review of progress in clinical photodynamic therapy," Technol. Cancer Res. Treat. 4, 283-293 (2005).

2. B. C. Wilson and M. S. Patterson, "The physics, biophysics, and technology of photodynamic therapy," Phys. Med. Biol. 53, R61-R109 (2008).

3. T. C. Zhu and J. C. Finlay, "The role of photodynamic therapy (PDT) physics," Med. Phys. 35, 3127-3136 (2008).

4. P. Agostinis et al., "Photodynamic therapy of cancer: an update," $C A$ Cancer J. Clin. 61, 250-281 (2011).

5. C. B. Simone et al., "Photodynamic therapy for the treatment of nonsmall cell lung cancer," J. Thorac. Dis. 4, 63-75 (2012).

6. B. W. Pogue et al., "Revisiting photodynamic therapy dosimetry: reductionist and surrogate approaches to facilitate clinical success," Phys. Med. Biol. 61, R57-R89 (2016).

7. M. M. Kim et al., "On the in vivo photochemical rate parameters for PDT reactive oxygen species modeling," Phys. Med. Biol. 62, R1-R48 (2017).

8. J. S. Friedberg et al., "Radical pleurectomy and intraoperative photodynamic therapy for malignant pleural mesothelioma," Ann. Thorac. Surg. 93(5), 1658-1667 (2012).

9. M. M. Kim et al., "PDT dose dosimeter for pleural photodynamic therapy," Proc. SPIE 9694, 96940Y (2016).

10. M. M. Kim, R. Penjweini, and T. C. Zhu, "Evaluation of singlet oxygen explicit dosimetry for predicting treatment outcomes of benzoporphyrin derivative monoacid ring A-mediated photodynamic therapy," J. Biomed. Opt. 22(2), 028002 (2017).

11. R. Penjweini et al., "Evaluation of the 2-(1-hexyloxyethyl)-2-devinyl pyropheophorbide (HPPH) mediated photodynamic therapy by macroscopic singlet oxygen modeling," J. Biophotonics 9(11-12), 1344-1354 (2016).

12. H. Qiu et al., "Macroscopic singlet oxygen modeling for dosimetry of Photofrin-mediated photodynamic therapy: an in-vivo study," J. Biomed. Opt. 21(8), 088002 (2016).

13. K. K. Wang et al., "Explicit dosimetry for photodynamic photodynamic therapy," J. Biophotonics 3(5-6), 304-318 (2010).

14. T. H. Foster et al., "Oxygen consumption and different effects in photodynamic therapy," Radiant. Res. 126, 296-303 (1991).

15. T. H. Foster, S. L. Gibson, and L. Gao, "Analysis of photochemical oxygen consumption effects in photodynamic therapy," Proc. SPIE 1646, 104-114 (1992).

16. T. H. Foster and L. Gao, "Dosimetry in photodynamic therapy-oxygen and the critical importance of capillary density," Radiat. Res. 130, 379-383 (1992)

17. L. Georgakoudi, M. G. Nichols, and T. H. Foster, "The mechanism of photfrin photobleaching and its consequences for photodynamic therapy," Photochem. Photobiol. 65, 135-144 (1997).

18. M. T. Jarvi, M. S. Patterson, and B. C. Wilson, "Insights into photodynamic therapy dosimetry: simultaneous singlet oxygen luminescence and photosensitizer photobleaching measurements," Biophys. J. 102, 661-671 (2012) 
19. M. Niedre, M. S. Patterson, and B. C. Wilson, "Direct near-infrared luminescence detection of singlet oxygen generated by photodynamic therapy in cells in vitro and tissues in vivo," Photochem. Photobiol. 75, 382-391 (2002).

20. I. Yuan et al., "Predictions of mathematical models of tissue oxygenation and generation of singlet oxygen during photodynamic therapy," Radiat. Res. 148, 386-394 (1997).

21. K. Beeson, E. Parilov, and M. Potasek, "Overview of computational simulations for PDT treatments based on optimal choice of singlet oxygen," Proc. SPIE 10047, 100470R (2017).

22. E. Oakley et al., "A new finite element approach for near real-time simulation of light propagation in locally advanced head and neck tumors," Lasers Surg. Med. 47, 60-67 (2015).

23. G. Gal Shafirstein et al., "Interstitial photodynamic therapy-focused review," Cancers 9, 12 (2017).

24. A. Johansson et al., "Realtime light dosimetry software tools for interstitial photodynamic therapy of the human prostate," Med. Phys. 34, 4309-4321 (2007).

25. A. Johansson et al., "Interstitial photodynamic therapy for primary prostate cancer incorporating realtime treatment dosimetry," Proc. SPIE 6427, 642700 (2007).

26. J. Swartling et al., "System for interstitial photodynamic therapy with online dosimetry: first clinical experiences of prostate cancer," J. Biomed. Opt. 15, 058003 (2010).

27. M. D. Altschuler et al., "Optimized interstitial PDT prostate treatment planning with the Cimmino feasibility algorithm," Med. Phys. 32, 3524-3536 (2005).

28. G. Yu et al., "Real-time in situ monitoring of human prostate photodynamic therapy with diffuse light," Photochem. Photobiol. 82, 1279-1284 (2006).

29. S. R. H. Davidson et al., "Treatment planning and dose analysis for interstitial photodynamic therapy of prostate cancer," Phys. Med. Biol. 54, 2293-2313 (2009).

30. D. C. Stripp, "Phase I trial of motexafin-lutetium-mediated interstitial photodynamic therapy in patients with locally recurrent prostate cancer," Proc SPIE 5315, 88-99 (2004).

31. K. Verigos et al., "Updated results of a phase I trial of motexafin lutetium-mediated interstitial photodynamic therapy in patients with locally recurrent prostate cancer," J. Environ. Pathol. Toxicol. Oncol. 25, 373-388 (2006).

32. H. Patel et al., "Motexafin lutetium-photodynamic therapy of prostate cancer: short and long term effect on prostate-specific antigen," Clin. Cancer Res. 14, 4869-4876 (2008).

33. J. Li and T. C. Zhu, "Determination of in-vivo light fluence distribution in heterogeneous prostate during photodynamic therapy," Phys. Med. Biol. 53, 2103-2114 (2008).

34. J. Li et al., "Integrated light dosimetry system for prostate photodynamic therapy," Proc. SPIE 6846, 68450Q (2008).

35. K. K. Wang and T. C. Zhu, "Reconstruction of in-vivo optical properties for human prostate using interstitial diffuse optical tomography," Opt. Express. 17(14), 11665-11672 (2009).

36. X. Liang, K. K. Wang, and T. C. Zhu, "Feasibility of interstitial diffuse optical tomography using cylindrical diffusing fiber for prostate PDT," Phys. Med. Biol. 58, 3461-3480 (2013).

37. T. C. Zhu, J. C. Finlay, and S. M. Hahn, "Determination of the distribution of light, optical properties, drug concentration, and tissue oxygenation in-vivo in human prostate during motexafin lutetium-mediated photodynamic therapy," J. Photochem. Photobiol. B 79, 231-241 (2005).

38. J. Li et al., "Optimization of light source parameters in the photodynamic therapy of heterogeneous prostate," Phys. Med. Biol. 53, 4107-4121 (2008).
39. T. C. Zhu et al., "A heterogeneous optimization algorithm for reacted singlet oxygen for interstitial PDT," Proc. SPIE 7551, 75510E (2010).

40. A. Yassine et al., "Automatic interstitial photodynamic therapy planning via convex optimization," Biomed. Opt. Express 9(2), 898-920 (2018).

41. L. Wang, S. L. Jacques, and L. Zheng, "MCML—Monte Carlo modeling of light transport in multi-layered tissues," Comput. Methods Programs Biomed. 47, 131-146 (1995).

42. J. Cassidy, V. Betz, and L. Lilge, "Treatment plan evaluation for interstitial photodynamic therapy in a mouse model by Monte Carlo simulation with FullMonte," Front. Phys. 3, 1-10 (2015).

43. Q. Fang and D. A. Boas, "Monte carlo simulation of photon migration in 3D turbid media accelerated by graphics processing units," Opt. Express 17, 20178-20190 (2009).

44. M. M. Kim et al., "Explicit macroscopic singlet oxygen modeling for benzoporphyrin derivative monoacid ring A (BPD)-mediated photodynamic therapy," J. Photochem. Photobiol B 164, 314-322 (2016).

45. T. C. Zhu et al., "Macroscopic modeling of the singlet oxygen production during PDT," Proc. SPIE 6427, 642708 (2007).

46. J. C. Finlay et al., "Diffuse reflectance spectra measured in vivo in human tissues during Photofrin-mediated pleural photodynamic therapy," Proc. SPIE 6139, 613900 (2006).

47. T. M. Busch et al., "Fluence rate-dependent intratumor heterogeneity in physiologic and cytotoxic responses to photofrin photodynamic therapy," Photochem. Photobiol. Sci. 8(12), 1683-1693 (2009).

48. T. C. Zhu, A. Lu, and Y. Ong, "An improved analytic function for predicting light fluence rate in circular fields on a semi-infinite geometry," Proc. SPIE 9706, 97061D (2016).

Karl W. Beeson received his PhD in physics from the University of Illinois at Urbana-Champaign. He is a cofounder and vice president for engineering at Simphotek, Inc. Previously, he cofounded Goldeneye Inc., and also worked in research and development organizations at Corning and Honeywell. His research interests include PDT dosimetry, optical devices, biomedical devices, and laser applications.

Evgueni Parilov received his $\mathrm{PhD}$ in computer science at New York University. $\mathrm{He}$ is a cofounder and chief technology officer at Simphotek, Inc. He is an expert in mathematical modeling and software development and is the chief software architect and a creator of SimphoSOFT ${ }^{\circledR}$. Previously, he worked at Courant Institute of Applied Mathematics.

Mary Potasek received her PhD in physics from the University of Illinois at Urbana-Champaign. She is a cofounder and chief scientific officer at Simphotek, Inc. She previously worked at Columbia University, New York University, Bell Laboratories, and the Air Force Research Laboratory. She is an expert in optical and lowtemperature experiments and numerical simulations. She is a senior member of IEEE, a SPIE fellow, and a recipient of an Outstanding Achievement Award from Bell Laboratories.

Michele M. Kim received her PhD in physics from the University of Pennsylvania in 2017 and is currently a medical physics resident at the Hospital of the University of Pennsylvania. Her research interests include preclinical and clinical photodynamic therapy dosimetry.

Timothy C. Zhu received his PhD in 1991 in physics from Brown University. $\mathrm{He}$ is currently a professor in the Department of Radiation Oncology at the University of Pennsylvania. His current research interests include explicit PDT dosimetry, singlet oxygen explicit dosimetry (SOED), integrated systems for interstitial and intracavitary PDT, diffuse optical tomography, in vivo dosimetry, and external beam radiation transport. 